\title{
Infant Feeding Practices in the First Year of Life in a Metropolitan Italian Cohort
}

\author{
Giulia Cinelli ${ }^{1}$, Marta Fabrizi ${ }^{1}$, Blegina Shashaj ${ }^{1}$, Giuseppe De Matteis ${ }^{1,2}$, Giorgio Bedogni ${ }^{3}$, \\ Donatella Comparcola $^{4}$, Stefania Ruggeri ${ }^{5}$, Valerio Nobili ${ }^{4}$, Melania Manco ${ }^{1, *}$ \\ ${ }^{1}$ Research Unit for Multifactorial Disease, Bambino Gesù Children's Hospital, IRCCS (Istituto di Ricovero e Cura a Carattere \\ Scientifico), viale di San Paolo 15, Rome 00146, Italy \\ ${ }^{2}$ Department of Internal and Emergency Medicine, Catholic University of the Sacred Heart, Rome, Italy \\ ${ }^{3}$ Clinical Epidemiology Unit, Liver Research Center Basovizza, Trieste, Italy \\ ${ }^{4}$ Hepato-Metabolic Disease Unit, Bambino Gesù Children's Hospital and IRCCS, Rome, Italy \\ ${ }^{5}$ CREA- Alimenti e Nutrizione, Via Ardeatina, 54600178 Rome, Italy \\ *Corresponding author: melania.manco@opbg.net
}

\begin{abstract}
Infant feeding choices are pivotal for growth and may affect the risk of overweight later in life. The objective of the study was to explore: (i) adherence to World Health Organization (WHO, exclusive breast feeding, $\mathrm{BF}$, in the first 6 months) and European Society for Paediatric Gastroenterology Hepatology and Nutrition (ESPHGAN, complementary feeding, CF, between 17 and 26 weeks of age and cow's milk introduction at 1 year of age) recommendations; (ii) and the effect of these practice on infants' anthropometrics and adiposity at 1 year of age. The study was based on the sample of infants $(\mathrm{n}=462)$ from the "Feeding" cohort whose feeding behaviours were monthly investigated from birth to 1 year of age and anthropometrics, thickness of epicardial adipose (EAT), abdominal subcutaneous, and visceral adipose tissues, measured at 1 year of age. Exclusively breast-fed infants were $69.5 \%(n=321)$ at birth but $19.9 \%(n=92)$ at 6 months of age. CF was introduced at the median age of $5(4-6)$, with $64.5 \%(n=298)$ of infants complying the ESPHGAN guidelines, while cow's milk was introduced at the age of 11 (10-12) months. Age of CF was associated with weight-to-length ratio (coeff. $=-0.002,95 \%$ CI -0.003 to -0.000 , $\mathrm{p}=0.018, \mathrm{r}$ ). Age of cow's milk introduction with weight-to-length ratio as well (coeff. $=0.001,95 \% \mathrm{CI} 0.000$, $0.002, \mathrm{p}=0.025$; and EAT (coeff. $=0.038,95 \%$ CI 0.012 to $0.063, \mathrm{p}=0.004$ ) at $1-\mathrm{y}$. Feeding practices complied with WHO recommendations in one infant out of five, and ESPHGAN recommendations in about seven out of ten. Early CF and cow's milk were both associated with anthropometrics at 1-y, while exclusively BF was not.
\end{abstract}

Keywords: infant diet, breastfeeding, formula feeding, complementary feeding, growth, visceral adiposity

Cite This Article: Giulia Cinelli, Marta Fabrizi, Blegina Shashaj, Giuseppe De Matteis, Giorgio Bedogni, Donatella Comparcola, Stefania Ruggeri, Valerio Nobili, and Melania Manco, "Infant Feeding Practices in the First Year of Life in a Metropolitan Italian Cohort." Journal of Food and Nutrition Research, vol. 6, no. 2 (2018): 82-88. doi: 10.12691/jfnr-6-2-3.

\section{Introduction}

Infant feeding choices during the first year of life are pivotal for infants' growth. Growth pattern during the first 12 months of life and early excessive weight gain are associated with later adverse health outcomes [1] such as overweight and increased body fat deposition [2,3].

Within this time-frame, two milestones of human dietary behaviours have been identified to be associated with paediatric obesity: (1) breastfeeding (BF) vs. formula feeding (FF) and (2) timing and pattern of complementary feeding (CF) [4,5]. The World Health Organization (WHO) recommends for the general population exclusive breastfeeding until 6 months of age, after which BF should continue but appropriate complementary foods should be introduced in order to meet the nutritional requirements of the child [6]. Indeed, CF, solid foods and liquids other than breast milk or infant formula and follow-on formula, is defined as the transition from breast milk to the family diet [6]. As regards solid foods, the European Society for Paediatric Gastroenterology Hepatology and Nutrition (ESPGHAN) recommends the introduction of solid foods not earlier than 17 weeks (beginning of the 5th month of life) and not later than 26 weeks (beginning of the 7th month). Nevertheless, ESPGHAN suggests also that cow's milk, being a poor source of iron, should not be the main drink before 12 months of age (although small volumes may be added to complementary foods) [7].

A recent meta-analysis, comparing breast-fed with nonbreast-fed infants, showed the former to be associated with $15 \%$ lower odds of childhood overweight than nonbreast-fed ones [3]. The most widely accepted explanation of the protective effect of BF is the difference in child growth rate of breast vs. formula-fed infants [8,9]. As demonstrated by a longitudinal multicenter Randomized Control Trial [10] and by a national cohort study [11], breast-fed infants grow differently than formula-fed 
infants, with the latter having a higher weight and weight-for-length at $1-\mathrm{y}$ of age. Although BF, conversely to FF, is associated with a slightly lower BMI in childhood and adulthood, a precise assessment of the preventive role of BF is difficult to perform because of the many confounding factors [12].

Early CF may represent a risk factor for childhood obesity, even though the relationship between the timing of food introduction and obesity is not straightforward [5]. Early introduction of solid foods is associated with young maternal age, low maternal education, formula feeding, multiparity, shorter breastfeeding duration and higher maternal BMI $[13,14]$.

The objective of the present study was to investigate infant feeding practices during the first year of life in a cohort of infants from a metropolitan area of Central Italy. In detail, we investigated (i) adherence to $\mathrm{WHO}$ and ESPGHAN recommendations for $\mathrm{BF}$ and timing of $\mathrm{CF}$ and cow's milk introduction; (ii) association of $\mathrm{BF}, \mathrm{FF}$ and timing of CF and cow's milk with anthropometrics and adiposity at 1-year of age.

\section{Materials and Methods}

\subsection{Subject and Study Design}

The "Feeding Low-Grade Inflammation and Insulin Resistance of the Foetus" project is a prospective cohort study of 1000 mother-infant pairs. Its primary aim was to evaluate the association at birth between maternal erythrocyte concentrations of fatty acids (FAs) and the infant's insulin resistance and low-grade inflammation. Details of the study are given elsewhere [15]. Eight hundred forty-seven mothers completed the study giving birth. At birth, newborns were enrolled in an ancillary study aimed at investigating the association between in utero exposure to maternal FAs and growth and adiposity at 1 year of age.

Eligibility criteria for the study were: gestational age between 37th and 42nd week, Apgar score greater than 7 at five minutes and absence of genetic disorders.

The study was approved by the Ethical Committee of the "Ospedale Pediatrico Bambino Gesù" (OPBG), in agreement with national and international regulations and the Declaration of Helsinki (2000). All participants signed an informed consent.

\subsection{Enrolment Visit}

Newborns' anthropometrics (birth weight, BW; birth crown-heel length, BL and head circumference, HC) was measured at birth. BW was measured within 1 hour from delivery with an electronic weighing scale and recorded to the nearest $5 \mathrm{~g}$. BL and $\mathrm{HC}$ were measured within 1 day from delivery with a Harpenden neonatometer and an inelastic tape, respectively, and recorded to the nearest millimetre [16]. Information on parents' age, body weight, height, level of education (secondary school or lower, high school, university), profession (employed or unemployed), smoking and parity was recorded. Maternal pre-pregnancy anthropometry was self-reported. The body mass index (BMI) was calculated as $\mathrm{kg} / \mathrm{m} 2$ and classified according to the World Health Organization (WHO) [17]. Gestational weight gain $(\mathrm{GWG})$ was calculated by subtracting the prepregnancy weight to the weight reached at time of delivery and classified according to the Institute of Medicine (IOM) guidelines as adequate, inadequate or excessive $[15,18]$.

Mothers received a self-reported diary (food timetable) to investigate infants feeding practices during the whole first year of life of their infants. They were asked to monthly record the first introduction of each of the following food groups: milk (breast, formula, mixed, soy, rice, cow's), fruits, vegetables, cereals, meat, fish, dairy products, eggs, legumes, oil, sugars, honey, tea/chamomile tea/infusion, other beverages. They were invited to complete the diary with the support of the General Practitioner (GP).

\subsection{Follow-up Visit}

The diary was handed back to the researchers when the child was 12 months old, at the follow-up visit. During the visit the nutritionist (G.C.) interviewed parents using a questionnaire and the standard 24-hour recall method. The questionnaire investigated the same food groups of the food timetable.

Anthropometrics were measured during the follow-up visit, according to standardized procedures. Infants were weighed naked on an mechanical baby scale with sliding weights (SECA 745) accurate to $10 \mathrm{~g}$. Length was measured on a portable baby measuring rod with large callipers (SECA 07) accurate to $1 \mathrm{~mm}$, while $\mathrm{HC}$ was measured using a measuring inelastic tape. All measurements were performed by the same trained paediatrician.

Standard deviation scores (SDS) for infant weight and length were calculated using the World Health Organization (WHO) charts (Anthro software program for Windows, version 3.2.2). Infant growth was assessed as the difference (delta) between anthropometrics at 1 year of age and anthropometrics at birth.

\subsection{Ultrasound Assessment}

Ultrasound (US) assessment was independently performed by two experienced US operators using an ACUSON X700 Ultrasound System (Siemens Healthcare, Siemens AG, Munich, Germany). Each infant underwent (i) a transtoracic two-dimensional (2D) echocardiogram to measure epicardial adipose thickness (EAT) and (ii) an abdominal US to quantify abdominal fat distribution measuring the US-abdominal visceral adipose tissue (USVAT) and the US-abdominal subcutaneous adipose tissue (US-SAT) depths. Epicardial adipose thickness (EAT) was evaluated using a $1.0-4.0 \mathrm{MHz}$ Phased Array (Siemens Healthcare, Munich, Germany). Echocardiogram was performed with subjects in the supine position and EAT was measured on the free wall of the right ventricle, in parasternal long and short-axis views, during endsystole in 3 cardiac cycles. The echo-free area extending from epicardial surface to parietal pericardium in front of the right ventricular free wall was vertically measured at the thickest level, using aortic annulus and mid-chordal region as landmarks in long-axis and short axis view, 
respectively. US-VAT depth was measured with a $1.0-5.0 \mathrm{MHz}$ abdominal curved array transducer (Siemens Healthcare, Munich, Germany) while US-SAT depth was measured with a $5.0-10.0 \mathrm{MHz}$ wide bandwidth linear transducer (Siemens Healthcare, Munich, Germany). For both measures, the transducers were positioned where the xiphoid line intercepted the waist measurement plane, and the images were taken during expiration. US-VAT depth was measured on a longitudinal plane and was defined as the distance between the peritoneal border and the corpus of the lumbar vertebra. US-SAT depth was measured at the same location, but on transverse plane and was defined as the distance between the cutaneous border and the linea alba [19].

\subsection{Statistical Analysis}

Most continuous variables were not Gaussian-distributed and all are reported as medians and interquartile range (IQR). Categorical variables are reported as counts and proportions. Median regression for single and repeated measures was run to investigate effects of feeding practices on anthropometrics and adiposity. Statistical significance was set at $\mathrm{p} \leq 0.05$. Statistical analyses were performed using available software (STATA 12.1; Stata Corp LP).

\section{Results}

\subsection{Subjects}

The flow of the enrolled newborns is reported in Figure 1.

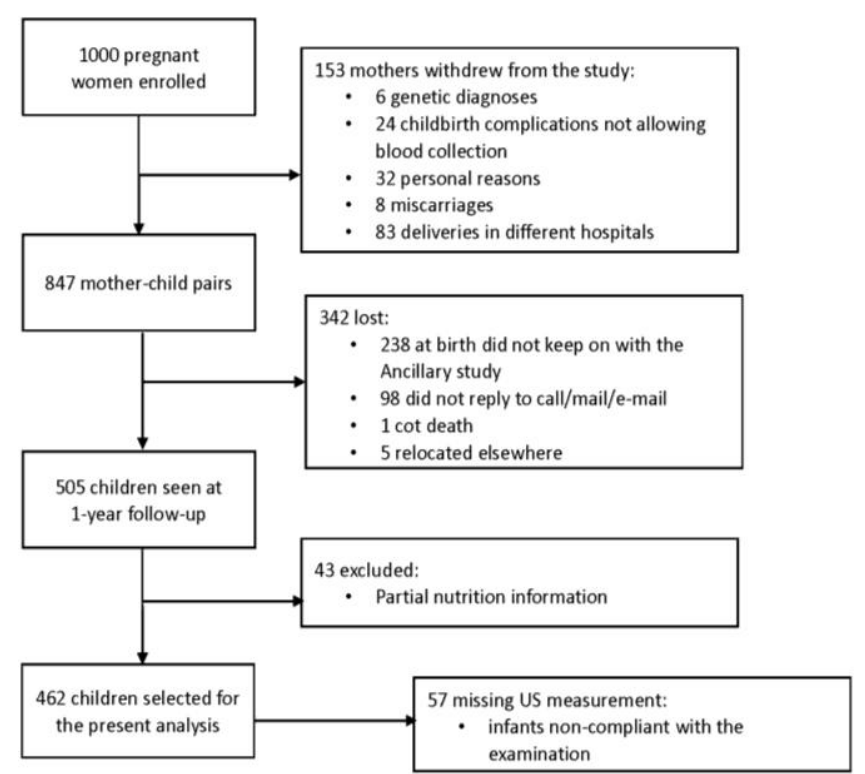

Figure 1. The figure represents the flowchart followed for the study sample's selection. The different reasons why the subjects have not been included in the analysis are described.

Four hundred and sixty-two mother-infant pairs, for which information about breastfeeding, complementary feeding and anthropometric measurements was available, were selected and used for the analysis of the present study. Due to lack of compliance with the examination, US assessment was carried out on a sub-sample of 405 infants. Table 1 shows mothers' and infants' characteristics.
Information about feeding practices was incomplete or not available in 43 out of the 505 infants (8.5\%) who completed the ancillary study. No difference was found in age, anthropometrics and Socioeconomic Status (SES) of women whose newborns were excluded from the present analysis (data not reported).

Table 1. Maternal and infant characteristics

\begin{tabular}{|c|c|c|}
\hline & Variable & $n=462$ \\
\hline \multirow[t]{25}{*}{ Mothers } & Age (years) & $34.0(30.0-38.0)^{1}$ \\
\hline & Pre-pregnancy BMI $\left(\mathrm{kg} / \mathrm{m}^{2}\right)$ & $22.03(19.9-24.7)^{1}$ \\
\hline & Underweight & $36(7.8)^{2}$ \\
\hline & Normal weight & $316(68.4)^{2}$ \\
\hline & Overweight & $80(17.3)^{2}$ \\
\hline & Obese & $30(6.5)^{2}$ \\
\hline & GWG & $13.0(10.0-16.0)^{1}$ \\
\hline & Inadequate GWG & $130(28.1)^{2}$ \\
\hline & Adequate GWG & $193(41.8)^{2}$ \\
\hline & Excessive GWG & $139(30.1)^{2}$ \\
\hline & Parity & \\
\hline & 0 & $250(54.2)^{2}$ \\
\hline & 1 & $171(37.1)^{2}$ \\
\hline & $\geq 2$ & $40(8.7)^{2}$ \\
\hline & Gestational age (weeks) & $39.0(38.0-40.0)^{1}$ \\
\hline & Delivery method & \\
\hline & Vaginal & $267(57.4)^{2}$ \\
\hline & Caesarean & $195(41.9)^{2}$ \\
\hline & Occupation & \\
\hline & Employed & $130(28.4)^{2}$ \\
\hline & Unemployed & $328(71.6)^{2}$ \\
\hline & Level of education & \\
\hline & Secondary school or lower & $51(11.1)^{2}$ \\
\hline & High school & $210(45.9)$ \\
\hline & University & $197(43.0)^{2}$ \\
\hline \multirow[t]{30}{*}{ Infants } & Infant sex: male & $237(51.3)^{2}$ \\
\hline & BW (g) & $3315.0(3020.0-3630.0)^{1}$ \\
\hline & $\mathrm{BL}(\mathrm{cm})$ & $50.0(49.0-52.0)^{1}$ \\
\hline & Head Circumference $(\mathrm{cm})$ & $35.0(34.0-36.0)^{1}$ \\
\hline & Weight-for-length & $0.07(0.06-0.07)$ \\
\hline & BW-for-age (SDS) & $0.08(-0.57-0.76)^{1}$ \\
\hline & BL-for-age (SDS) & $0.15(-0.40-1.06)^{1}$ \\
\hline & HC-for-age (SDS) & $0.69(-0.11-1.30)^{1}$ \\
\hline & Weight-for-length (SDS) & $-0.39(-1.01-0.23)^{1 *}$ \\
\hline & Classification & \\
\hline & SGA & $33(7.1)^{2}$ \\
\hline & AGA & $372(80.5)^{2}$ \\
\hline & LGA & $57(12.3)^{2}$ \\
\hline & Age at follow-up (days) & $380.0(371.0-401.0)^{1}$ \\
\hline & Weight 1-y (g) & $10000(9200-10810)^{1}$ \\
\hline & Length 1-y $(\mathrm{cm})$ & $76.5(75.0-79.0)^{1}$ \\
\hline & HC 1-y (cm) & $46.0(45.5-47.0)^{1}$ \\
\hline & BMI 1-y $\left(\mathrm{kg} / \mathrm{m}^{2}\right)$ & $16.91(16.06-18.08)^{1}$ \\
\hline & Weight-for-length & $0.13(0.12-0.14)^{1}$ \\
\hline & Weight-for-age 1-y (SDS) & $0.46(-0.15-1.11)^{1}$ \\
\hline & Length-for-age 1-y (SDS) & $0.27(-0.41-1.05)^{1}$ \\
\hline & HC-for-age 1-y (SDS) & $0.48(-0.150-1.08)^{1}$ \\
\hline & BMI 1-y (SDS) & $0.35(-0.27-1.05)^{1}$ \\
\hline & Weight-for-length 1-y (SDS) & $0.42(-0.20-1.03)^{1}$ \\
\hline & Delta weight (SDS) & $0.36(-0.38-1.04)^{1}$ \\
\hline & Delta length (SDS) & $0.00(-0.74-0.85)^{1}$ \\
\hline & Delta weight-for-length (SDS) & $0.7(-0.12-1.66)^{1} *$ \\
\hline & EAT (mm) & $3.4(3.10-3.60)^{1}$ \\
\hline & US-VAT (mm) & $45(43.00-47.00)^{1}$ \\
\hline & US-SAT (mm) & $3.6(3.10-3.93)^{1}$ \\
\hline
\end{tabular}

Data are expressed as the median $(\mathrm{IQR})^{1}$ or as a percentage $(\%)^{2}$; AGA adequate for gestational age; BMI, body mass index; BW, birth weight; BL, birth length; GWG, gestational weight gain; HC, head circumference; LGA, large for gestational age; SGA, small for gestational age. Anthropometric measures were taken at study enrolment for mothers, at birth and at 1-y for infants.

$* \mathrm{n}=459$ because BL was below $45.0 \mathrm{~cm}$ 
Table 2. Median regression between infant feeding choices and maternal educational level

\begin{tabular}{|c|c|c|c|c|c|c|}
\hline & \multicolumn{2}{|c|}{ BF duration } & \multicolumn{2}{|c|}{ Introduction of $\mathrm{CF}$} & \multicolumn{2}{|c|}{ Introduction of cow's milk } \\
\hline & Coeff. (95\% CI) & $\mathbf{p}$ & Coeff. (95\% CI) & $\mathbf{p}$ & Coeff. $(95 \%$ CI $)$ & $\mathbf{p}$ \\
\hline High school & $2.0(0.202,3.798)$ & 0.029 & $0.0(-0.534,0.534)$ & 1.000 & $-3.5(-9.837,2.837)$ & 0.278 \\
\hline Univ. & $3.0(0.981,5.019)$ & 0.004 & $0.0(-0.506,0.506)$ & 1.000 & $-9.0(-11.417,-6.583)$ & 0.000 \\
\hline Inter. & $3.0(1.747,4.253)$ & 0.000 & $6.0(5.549,6.451)$ & 0.000 & $9.0(6.745,11.255)$ & 0.000 \\
\hline
\end{tabular}

Median regression analysis between infant feeding choices (BF, CF, cow's milk) and maternal educational level. Continuous variables: BF duration, introduction of CF and introduction of cow's milk. Categorical variables: education level (Ref.: primary school or lower). BF, breastfeeding; CF, complementary feeding; Univ., university; Inter., intercept.

\subsection{Breast, Formula and Cow's Milk}

Starting from birth, 321 (69.5\%) infants received breast milk, $74(16.0 \%)$ were supplemented with formula and 67 $(14.5 \%)$ were exclusively formula-fed.

At 3, 6 and 12 months of age, 252 (54.5\%), 194 (42.0\%) and $96(20.8 \%)$ infants were still breast-fed without any formula supplementation, respectively. Overall, 92 (19.9\%) infants were breast-fed without introduction of solid foods for the first 6 months, complying with WHO recommendations. The median (IQR) duration of breastfeeding was 5 (0-11) months, considering the first year of life.

The median (IQR) age of cow's milk introduction was 11 (10-12) months of age, with 138 infants (29.9\%) introducing it before the first year of age, conversely to what recommended by ESPGHAN.

Maternal education was positively associated with BF duration (Table 2). As compared to women with lower school degree, the median BF duration was in fact 2.0 (95\% CI 0.2 to 3.7) and 3.0 (95\% CI 0.9 to 5.0) months longer among women with high school and university degrees, respectively.

No association was found with maternal occupation status (employed vs. unemployed mothers) and duration of BF. Formula was not associated with earlier weaning, but it was significantly associated to an earlier cow's milk introduction (Coeff. $=-9.0$, 95\% CI -10.2 to -7.8 months, $\mathrm{p}<0.001)$.

\subsection{Complementary feeding}

The median age of introduction of solid foods was 5 (4-6) months. A total of 133 (28.8\%) infants, started CF at 6 months of age or after, on time considering WHO recommendations but late considering the ESPHGAN ones. According to the latter, 298 (64.5\%) infants introduced solid foods between 4 and 6 months of age, while $31(6.7 \%)$ were given solid foods prematurely, before 4 months of age.

Timing of CF was found to be positively correlated to BF $(r=0.299, p=0.000)$ and to time of cow's milk introduction $(\mathrm{r}=0.231, \mathrm{p}=0.000)$. At the median regression analysis, no correlation was found with mother's age, educational level, and parity.

\subsection{Association of Feeding Choices during the First Year and Anthropometrics at the Follow-up}

Breast and formula fed infants showed no significant difference in adiposity and duration of BF was not associated with anthropometrics at 1 year.
Formula feeding was positively associated to length z-score at 1 year of age (coeff. $=0.34,95 \% \mathrm{CI} 0.05$ to 0.72 , $\mathrm{p}=0.026$ ). No association was found with adiposity's indexes.

Duration of cow's milk was also positively associated to length (coeff. $=0.25,95 \%$ CI 0.081 to $0.419, \mathrm{p}=0.004$ ) and weight-to-length ratio at 1 year of age (coeff. $=0.001$, $95 \%$ CI $0.000,0.002, \mathrm{p}=0.025)$. A positive correlation between earlier cow's milk introduction and EAT was found (coeff. $=0.038,95 \% \mathrm{CI} 0.012$ to $0.063, \mathrm{p}=0.004$ ).

Timing of introduction of $\mathrm{CF}$ was negatively associated to weight-to-length ratio at 1 year of age (coeff. $=-0.002$, $95 \% \mathrm{CI}-0.003$ to $-0.000, \mathrm{p}=0.018)$, to weight $-\mathrm{Z}$-score at 1 year of age (coeff. $=-0.129,95 \%$ CI -0.252 to -0.006 , $\mathrm{p}=0.040)$.

\section{Discussion}

Our findings show that about $70 \%$ of mothers from a metropolitan area start feeding their offspring exclusively with their milk but only one infant out of five is still breast-fed at 6 months of age, without introduction of solid food, as recommended by the WHO [6]. Mothers who breast-fed were more educated than mothers who opted for formula feeding. Conversely, maternal occupation (employed mothers vs. unemployed) was not related to the breastfeeding practice. In our series, figures of breast-fed infants were not different from prevalence described in a sample of 6,942 infants enrolled in two population-based follow-up surveys conducted by the Italian National Institute of Health in 2008/2009 and 2010/2011 [20]. The only exception was about the percentage of infants breast-fed for the first six months of life which was higher in our sample $(19.9 \%$ vs. $5.5 \%)$. Carletti et al. 2017 described feeding practices and compliance to WHO recommendation in 148 infants in Northern Italy between July 2007 and July 2008 and followed up for three years by the GPs. At six months of age only $7 \%$ of infants were exclusively breast-fed, unlike our population $(19.9 \%$ ), while a total of $33 \%$ of infants were still on exclusive or combined to $\mathrm{CF}$ breast milk, differently to what we found $(42 \%)$. Only $14 \%$ of infants were given their first complementary food at six months of age or more [21]. In contrast, in our sample $28.8 \%$ of infants started CF when they were 6-months-old or later, according to WHO recommendations.

The prospective European Childhood Obesity Project investigated feeding practices in 1,678 infants from 5 countries between 2002 and 2004, including the metropolitan area of Milan in Northern Italy. The researchers found $6.0 \%$ of FF infants, but only $0.6 \%$ of the 
BF infants, introducing CF already at the age of 3 months, while solids were introduced later than recommended by the ESPHGAN in $0.7 \%$ of FF infants and $2.3 \%$ of BF infants, respectively [22]. In our cohort, $6.7 \%(\mathrm{n}=31)$ and $28.8 \%(\mathrm{n}=133)$ of mothers started weaning practices earlier (before 4 months of age) or later (after 6 months of age), respectively. The majority of our population started $\mathrm{CF}$ feeding on time (between the beginning of the 5th month of life the beginning of the 7th month), complying with the ESPHGAN recommendations [7], and breastfeeding was related to a later introduction of solid foods.

Cow's milk was introduced in FF infants significantly earlier than BF infants and $29.9 \%$ of mothers introduced it before the infant was 1-year-old, while $70.1 \%$ met ESPHGAN recommendations. These results are in line with Carletti's findings with $63 \%$ of infants introducing cow's milk after 12 months [21].

Figures suggest an apparent improvement in the six-months exclusive $\mathrm{BF}$ and consequent later introduction of $\mathrm{CF}$ in breast-fed infants, when compared to the past [20,21].

We suppose that the higher percentages of breast-fed infants meeting the WHO recommendations in our survey reflects the improvement of feeding practices over the last 10 years in the general Italian population, probably as a consequence of our National Health Service's effort spent on the promotion of breastfeeding. Mothers were mostly educated ( $88.9 \%$ had secondary school degree or higher) but it is unlikely that this reflects a selection bias of the women enrolled in the study, since the degree of education was the same for those who did not consent (unpublished data).

The main aim of the "Feeding" study was to investigate the low-grade inflammation of the foetus in relation to maternal dietary intake of fatty acids. The present investigation was out of the scope of the study and aimed to describe infants feeding practices during the first year of life in a subsample of the cohort, and their relationship on anthropometric measures at 1-y. Differently to what described in literature by Agostoni and Koletzko, in 1999 and 2009 respectively, we did not find significant differences between the pattern of growth of breast-fed and formula-fed infants [10,11]. In our cohort, we found no association between infants' anthropometrics at 1 year and BF duration, while FF duration was found to be positively associated to length at 1 year. These results are in agreement with old studies showing that in formula-fed infants higher Insulin-like growth factor-I (IGF-I) concentrations may lead to faster gains in length [23]. Analogously, cow's milk was found to be positively correlated to infants' length and to weight-to-length ratio at 1-y. These results are in line with previous studies finding circulating IGF-I positively associated with intakes of animal protein and milk, and both IGF-I and milk associated to height [24]. Our results suggest that cow's milk, as well as FF and early complementary CF, may provide some growth-stimulating components affecting infant growth. Interestingly, cow's milk was also associated to the EAT, while no association was found with BF, FF or CF. The EAT shares a common embryological origin with the VAT thus it is classified as metabolically active adipose tissue and it is considered an accurate and practical estimate of visceral adiposity in infants [25]. Furthermore, the EAT measurement by echocardiography had demonstrated to significantly correlate, both in adults and in pre- and post- pubertal children with the intra-abdominal accumulation of visceral fat as measured on cardiac magnetic resonance imaging [26].

In conclusion, what was impressive is that breast feeding practices in infants are not still widespread in 2013-2015, even if an improvement was observed in this study. Massive policies and international claims promoting breastfeeding may have played a role in improving early infant feeding choices. Nevertheless, even if the percentages appeared to be significantly improved, nowadays only one infant out of 5 was exclusive breast-fed for six months, or was still breast-fed at 1 year of age, showing the partial compliance with WHO recommendations on $\mathrm{BF}$ and $\mathrm{CF}$ in a sample of metropolitan area of Central Italy. This could be related to different factors, mainly to the difference between ESPGHAN and WHO recommendations about solid food introduction that still exists [27].

The main limitations of the present study are the following: the exploration of infant feeding practices was out of the primary scope of the "Feeding" study so we were able to infer some information; anthropometrics were measured only at birth and at 1-y, self-reported measures were excluded since not always reliable; the heterogeneity of data collection prevented an appropriate comparison with previous studies.

\section{Conclusion}

In our series $\mathrm{BF}$ did not appear protective against excess weight gain at 1 year while early introduction of $\mathrm{CF}$ represented a statistically significant risk factor. Nevertheless, we can hypothesize BF to be protective against excess weight gain by delaying time of food introduction. Finally, early cow's milk introduction, before 12 months of age, may represent a risk factor for growth and visceral fat deposition.

Infant feeding during the first year of life represents a fundamental issue of medical and public health interventions to prevent or reduce the burden of childhood obesity. Women's empowerment on importance of exclusive breastfeeding for the first 6 months of life, as recommended by the $\mathrm{WHO}$, and campaigns to encourage it during the whole first year of life should be increased. More awareness about adequate complementary feeding practices, in terms of timing and introduction of cow's milk is needed and should be translated into specific National Guidelines, shared among GPs.

\section{Acknowledgements}

We thank the parents who took part the study.

\section{Funding}

This study was supported by a research grant from the Italian Ministry of Health to MM (GR-2010 2304957 RC 201602P003767). The funder had no role in the design 
of the study; in the collection, analyses, or interpretation of data; in the writing of the manuscript, and in the decision to publish the results.

\section{Statement of Conflict of Interest}

The authors declare no conflict of interest.

\section{Abbreviations}

$\begin{array}{ll}\text { BF } & \text { breastfeeding } \\ \text { BL } & \text { birth crown-heel length } \\ \text { BMI } & \text { body mass index } \\ \text { BW } & \text { birth weigh } \\ \text { CF } & \text { complementary feeding } \\ \text { CHOP } & \text { childhood obesity program } \\ \text { EAT } & \text { epicardial adipose thickness } \\ \text { ESPHGAN } & \text { european society for paediatric } \\ & \text { gastroenterology hepatology and nutrition } \\ \text { FAs } & \text { fatty acids } \\ \text { FF } & \text { formula feeding } \\ \text { GDM } & \text { gestational diabetes mellitus } \\ \text { GP } & \text { general practitioner } \\ \text { HC } & \text { head circumference } \\ \text { IGF-I } & \text { insulin-like growth factor-I } \\ \text { IOM } & \text { institute of medicine } \\ \text { OPBG } & \text { ospedale pediatrico Bambino Gesù } \\ & \text { (Bambino Gesù children hospital) } \\ \text { RCT } & \text { randomized controlled trial } \\ \text { SD } & \text { standard deviation } \\ \text { SDS } & \text { standard deviation score } \\ \text { SES } & \text { socioeconomic status } \\ \text { US } & \text { ultrasound } \\ \text { US-SAT } & \text { US-abdominal subcutaneous adipose } \\ \text { US-VAT } & \text { tissue } \\ \text { WHO } & \text { world health organization } \\ & \end{array}$

\section{References}

[1] Koletzko, B. (2005). "Early nutrition and its later consequences: new opportunities", Advances in Experimental Medicine and Biology, 569. 1-12. 2005.

[2] Stettler, N, "Nature and strength of epidemiological evidence for origins of childhood and adulthood obesity in the first year of life." International journal of obesity, 31 (7). 1035-1043. July 2007.

[3] Weng, S. F., Redsell, S. A., Swift, J. A., Yang, M., Glazebrook, C. $\mathrm{P}$, "Systematic review and meta-analyses of risk factors for childhood overweight identifiable during infancy", Archives of disease in childhood, 97(12). 1019-1026. December 2012

[4] Arenz, S., Rückerl, R., Koletzko, B., \& von Kries, R, "Breastfeeding and childhood obesity-a systematic review", International journal of obesity, 28(10). 1247-1256. October 2004.

[5] Pearce, J., Taylor, M. A., \& Langley-Evans, S. C, "Timing of the introduction of complementary feeding and risk of childhood obesity: a systematic review", International journal of obesity, 37(10). 1295-1306. October 2013.

[6] WHO. World Health Organization, UNICEF, Global strategy for infant and young child feeding, World Health Organization, 2003. [Online] Available:

whqlibdoc.who.int/publications/2003/9241562218.pdf [Accessed: Dec. 12, 2017]].
[7] Fewtrell, M., Bronsky, J., Campoy, C., Domellöf, M., Embleton, N., Mis, N. F., Hojsak, I., Hulst, J.M., Indrio, F., Lapillonne, A., Molgaard, C, "Complementary feeding: A position paper by the European Society for Paediatric Gastroenterology, Hepatology, and Nutrition (ESPGHAN) committee on nutrition", Journal of pediatric gastroenterology and nutrition, 64(1). 119-132. January 2017.

[8] Koletzko, B., von Kries, R., Monasterolo, R. C., Subías, J. E., Scaglioni, S., Giovannini, M., Beyer, J., Demmelmair, H., Anton, B., Gruszfeld, D., Dobrzanska, A, "Can infant feeding choices modulate later obesity risk?", The American journal of clinical nutrition, 89(5). 1502S-1508S. May 2009.

[9] Singhal, A., Kennedy, K., Lanigan, J., Fewtrell, M., Cole, T. J., Stephenson, T., Elias-Jones, A., Weaver, L.T., Ibhanesebhor, S., MacDonald, P.D., Bindels, J., Lucas, A, "Nutrition in infancy and long-term risk of obesity: evidence from 2 randomized controlled trials", The American journal of clinical nutrition, 92(5). 1133-1144. November 2010.

[10] Koletzko, B., von Kries, R., Closa, R., Escribano, J., Scaglioni, S., Giovannini, M., Beyer, J., Demmelmair, H., Gruszfeld, D., Dobrzanska, A., Sengier, A., Langhendries, J.P., Rolland Cachera, M.F., Grote, V, "Lower protein in infant formula is associated with lower weight up to age 2 y: a randomized clinical trial", The American journal of clinical nutrition, 89(6). 1836-1845. June 2009.

[11] Agostoni, C., Grandi, F., Gianni, M. L., Silano, M., Torcoletti, M., Giovannini, M.., Riva, E, "Growth patterns of breast fed and formula fed infants in the first 12 months of life: an Italian study", Archives of disease in childhood, 81(5). 395-399. November 1999.

[12] Owen, C. G., Martin, R. M., Whincup, P. H., Davey-Smith, G., Gillman, M. W., Cook, D. G, "The effect of breastfeeding on mean body mass index throughout life: a quantitative review of published and unpublished observational evidence", The American journal of clinical nutrition, 82(6). 1298-1307. December 2005.

[13] Schrempft, S., van Jaarsveld, C. H., Fisher, A., Wardle, J, "Family and infant characteristics associated with timing of core and non-core food introduction in early childhood", European journal of clinical nutrition, 67(6). 652-657. June 2013.

[14] Tromp, I. I. M., Briede, S., Kiefte-de Jong, J. C., Renders, C. M., Jaddoe, V. W. V., Franco, O. H., Hofman, A., Raat, H., Moll, H.A "Factors associated with the timing of introduction of complementary feeding: the Generation R Study", European journal of clinical nutrition, 67(6). 625-630. June 2013.

[15] Cinelli, G., Fabrizi, M., Ravà, L., Ciofi degli Atti, M., Vernocchi, P., Vallone, C., Pietrantoni, E., Lanciotti, R., Signore, F., Manco, $\mathrm{M}$, "Influence of maternal obesity and gestational weight gain on maternal and foetal lipid profile", Nutrients, 8(6). 368. June 2016.

[16] Bertino, E., Spada, E., Occhi, L., Coscia, A., Giuliani, F., Gagliardi, L., Gilli, G., Bona, G., Fabris, C., De Curtis, M., Milani, S, "Neonatal anthropometric charts: the Italian neonatal study compared with other European studies", Journal of pediatric gastroenterology and nutrition, 51(3). 353-361. September 2010.

[17] WHO. World Health Organization, Obesity: preventing and managing the global epidemic (WHO technical report series No. 894), World Health Organization, 2000. [Online] Available: whqlibdoc.who.int/trs/WHO_TRS_894.pdf [Access Dec. 12, 2017].

[18] IOM (Institute of Medicine) and NRC (National Research Council), Weight Gain During Pregnancy: Reexamining the Guidelines, The National Academies Press, 2009.

[19] De Lucia Rolfe, E., Modi, N., Uthaya, S., Hughes, I. A., Dunger, D. B., Acerini, C., Stolk, R.P., Ong, K. K, "Ultrasound estimates of visceral and subcutaneous-abdominal adipose tissues in infancy", Journal of obesity. 951-954. April 2013.

[20] Lauria, L., Spinelli, A., Grandolfo, M, "Prevalence of breastfeeding in Italy: a population based follow-up study", Annali dell'Istituto superiore di sanita, 52(3). 457-461. July-September 2016.

[21] Carletti, C., Pani, P., Monasta, L., Knowles, A., Cattaneo, A, "Introduction of Complementary Foods in a Cohort of Infants in Northeast Italy: Do Parents Comply with WHO Recommendations?", Nutrients, 9(1). 34. January 2017.

[22] Schiess, S., Grote, V., Scaglioni, S., Luque, V., Martin, F., Stolarczyk, A., Stolarczyk, A., Vecchi, F., Koletzko, B., European Childhood Obesity Project, "Introduction of complementary feeding in 5 European countries", Journal of pediatric gastroenterology and nutrition, 50(1). 92-98. January 2010. 
[23] Ong, K. K., Langkamp, M., Ranke, M. B., Whitehead, K., Hughes, I. A., Acerini, C. L., Dunger, D. B, "Insulin-like growth factor I concentrations in infancy predict differential gains in body length and adiposity: the Cambridge Baby Growth Study", The American journal of clinical nutrition, 90(1). 156-161. July 2009.

[24] Hoppe, C., Udam, T. R., Lauritzen, L., Mølgaard, C., Juul, A., Michaelsen, K. F, "Animal protein intake, serum insulin-like growth factor I, and growth in healthy 2.5-y-old Danish children", The American journal of clinical nutrition, 80(2). 447-452. August 2004.

[25] Iacobellis, G., Assael, F., Ribaudo, M. C., Zappaterreno, A. Alessi, G., Mario, U., Leonetti, F, "Epicardial fat from echocardiography: a new method for visceral adipose tissue prediction", Obesity, 11(2). 304-310. February 2003.

[26] Manco, M., Morandi, A., Marigliano, M., Rigotti, F., Manfredi, R., Maffeis, C, "Epicardial fat, abdominal adiposity and insulin resistance in obese pre-pubertal and early pubertal children", Atherosclerosis, 226(2). 490-495. February 2013.

[27] Cattaneo, A., Williams, C., Pallás - Alonso, C. R., Hernández Aguilar, M. T., Lasarte - Velillas, J. J., Landa - Rivera, L., Rouw, E., Pina, M., Volta, A., Oudesluys-Murphy, A.M, "ESPGHAN's 2008 recommendation for early introduction of complementary foods: how good is the evidence?", Maternal \& child nutrition, 7(4), 335-343. October 2011. 